$63^{\text {ème }}$ Congrès de la SFCO, 02024 (2015)

DOI:10.1051/sfco/20156302024

(C) Owned by the authors, published by EDP Sciences, 2015

\title{
COMMUNICATION
}

\section{Voie d'abord cervicale de dents de sagesse mandibulaires incluses : $\underline{\text { à propos de deux cas }}$}

\author{
Makhoul R*, Delbet-Dupas C**,***, Even $\mathrm{J}^{*, * * * *}$, Melka AC**, Barthelemy I**,***, \\ Mondié JM**,***, Pham-Dang $\mathrm{N}^{* * * * * *, * * * * *}$, Devoize $\mathrm{L}^{*, * * * * * * * * * *}$ \\ * Service d'Odontologie, CHU, Clermont-Ferrand \\ ** Service de Chirurgie Maxillo-Faciale, CHU, Clermont Ferrand \\ *** Faculté de Médecine, Université d'Auvergne, Clermont Ferrand \\ **** Faculté de Chirurgie dentaire, Université d'Auvergne, Clermont-Ferrand \\ ***** UMR Inserm/UdA, U1107, Neuro-Dol, Trigeminal Pain and Migraine, Faculté de Chirurgie dentaire, \\ Clermont-Ferrand
}

Les techniques chirurgicales d'avulsion des dents de sagesse sont très bien décrites et codifiées dans la littérature. Les chirurgiens oraux bénéficient ainsi d'un large arsenal de thérapeutiques et de méthodes afin d'aborder les troisièmes molaires mandibulaires incluses. Cependant, la voie d'abord intra-orale classique n'est plus suffisante en cas d'inclusion atypiqueet/ou d'accès limité.

Un homme de 42 ans a ainsi été adressé par son chirurgien dentiste traitant pour l'avulsion de 38. La radiographie panoramique a mis en évidence une troisième molaire profondément incluse et horizontale associée à une zone radioclaire péri-coronaire se référant probablement au développement d'un kyste dentigère. La tomodensitométrie (TDM) a précisé une position linguale des racines ; la furcation étant en relation intime avec le nerf alvéolaire inférieur (NAI). Devant les risques de développement du kyste et des complications possibles associées, l'indication chirurgicale a été posée. Un abord cervical par voie de sous maxillectomie a été retenu, l'abord endo-buccalnécessitant de réaliser une ostéotomie trop importante et de probablement sacrifier le NAI. Aucune séquelle postopératoire n'est à noter.

Le cas d'une patiente de 65 ans est également rapporté, adressée en urgence dans un contexte de tuméfaction mandibulaire post traumatique. L'examen cliniquea objectivé une paralysie faciale et une limitation permanente de l'ouverture buccale (LPOB) à $15 \mathrm{~mm}$, toutes deux conséquentes de la prise en charge chirurgicale d'un neurinome de l'acoustique en 2003. A l'examen endo-buccal, une suppuration en distal de la deuxième molaire était présente. Le cliché panoramique et la TDM ont mis en évidence une troisième molaire incluse ainsi qu'un trait de fracture entre celle-ci et la deuxième molaire. La même voie d'abord cervicale a été choisie, ici principalement en raison de la LPOB. L'avulsion des dents a été réalisée, la fracture réduite par mini-plaques d'ostéosynthèse. Les suites post-opératoires ont été simples, il n’y a pas eu d'accentuation des signes neurologiques.

Ces deux situations cliniques synthétisent les 3 indications de voie d'abord cervicales pour l'avulsion des dents de sagesse mandibulaires : 1/ la présence d'une dent incluse en position basilaire ou ectopique (Payne, Dickensen et Patel 2012) 2/ allant souvent depair, une position de la dent médiale ou inférieure au niveau du canal du NAI (Abu-El Naaj 2010, Ahmed and Speculand 2012, Dorochenko Martins et al. 2012) et 3/ une ouverture buccale insuffisante (Christensen 1950).

This is an Open Access article distributed under the terms of the Creative Commons Attribution License 4.0, which permits unrestricted use, distribution, and reproduction in any medium, provided the original work is properly cited. 
Le manque de visibilité dans une région difficilement abordable peut faire préférer une approche cervicale. Le risque majeur de ce type d'intervention est la lésion du rameau mentonnier du nerf facial qui longe le rebord basilaire mandibulaire. L'indication doit donc être murement réfléchie et rester une exception (Pippi 2005).

Nom et adresse du conférencier

Laurent DEVOIZE

Service d'odontologie CHU Estaing

1 place Lucie Aubrac

63000 Clermont Ferrand (France)

laurent.devoize@udamail.fr 